\title{
Análisis coste-efectividad del uso de espironolactona en el tratamiento de la insuficiencia cardiaca crónica
}

\author{
J. SOTO ÁLVAREZ, F. GONZÁLEZ VILCHEZ* \\ Unidad de Farmacoeconomía e Investigación de Resultados en Salud. Departamento de \\ Medicina. Pharmacia S.A. Madrid \\ *Sección de Cardiología. Servicio de Medicina Interna. Hospital General Yagüe. Burgos.
}

COST-EFFECTIVENESS OF THE USE OF SPIRONOLACTONE IN THE TREATMENT OF CHRONIC HEART FAILURE

\section{RESUMEN}

Objetivo: La insuficiencia cardiaca crónica (ICC) es la primera causa de hospitalización en personas mayores de 65 años. El uso de espironolactona en pacientes con ICC (grado III-IV) reduce su mortalidad y la tasa de hospitalización. El objetivo de este estudio ha sido evaluar la eficiencia del uso de espironolactona en el tratamiento de la ICC cuando se compara con el tratamiento convencional sólo.

Material y Métodos: El estudio se ha efectuado a través de un modelo farmacoeconomico basado en un análisis de decisión. Se ha realizado un análisis coste-efectividad a través de la simulación del comportamiento de dos hipotéticas cohortes de 1000 pacientes con ICC, habiéndose evaluado el coste de cada año de vida ganado con cada opción en estudio. Los datos incluidos han sido obtenidos de Rales, y se ha considerado que la supervivencia de los pacientes con ICC es de 4,3 años.

Solamente se han tenido en cuenta los costes directos médicos en el análisis, la perspectiva elegida ha sido la de la sociedad y el horizonte temporal ha sido de 4,3 años.

Resultados: La relación coste/efectividad fue mejor en el grupo espironolactona (1.961.214) que en el grupo de tratamiento estándar (2.242.912), y cada año extra de vida ganado al añadir espironolactona al tratamiento convencional supone un coste adicional de 591.457 ptas.

El análisis de sensibilidad efectuado, ha demostrado que la opción de usar espironolactona presenta siempre una mejor relación coste/efectividad, incluso en los peores escenarios.

Conclusiones: La asociación de $25 \mathrm{mg} /$ día de espironolactona al tratamiento convencional de la ICC, va a generar años de vida extra para los pacientes a un coste discreto, y perfectamente asumible por nuestro Sistema Nacional de Salud.

PALABRAS CLAVE: Insuficiencia cardiaca crónica. Espironolactona. análisis coste-efectividad. Años de vida ganados.

\section{ABSTRACT}

Background: Chronic heart failure (CHF) is the first cause of hospita lization in elderly patients. The use of spironolactone in patients with CHF (degree III-IV) reduces their mortality and the rate of hospitalization.

The aim of this study has been to assess the efficiency of using spiro nolactone in the treatment of CHF when compared with the use of conventional treatment alone.

Material and methods: The study has been performed through the design of a decision analytic model. A cost-effectiveness analysis was carried out by doing a simulation of 2 hypothetic cohorts of 1000 patients with CHF. It was evaluated the costs of each extra life year gai ned with each alternative under study.

Data included have been obtained from Rales and the mean survival time for these patients has been considered of 4.3 years.

Only direct medical costs have been included into this analysis. The chosen perspective has been the society and the time horizon included has been of 4.3 years.

Results: The cost/effectiveness ratio has been better in the spirono lactone group $(1,961,214)$ than in the standard treatment alone $(2,242,912)$ and each extra life year gained when adding spironolactone to the conventional treatment yielded an additional cost of 591,457 ptas.

The sensitivity analysis performed has shown that the option of using spironolactone presents always a better cost/effectiveness ratio even in the worst scenarios.

Conclusions: The association of $25 \mathrm{mg} /$ day of spironolactone to the conventional treatment of $\mathrm{CHF}$ is going to get more life years gained for these patients with a reasonable cost and clearly affordable by our National Health Service.

KEY WORDS: Chronic heart failure. Spironolactone. Cost/effectiveness analysis. Life years gained.

Soto Álvarez, J, González Vilchez. F. Análisis coste-efectividad del uso de espironolactona en el tratamiento de la insuficiencia cardiaca crónica. An Med Interna (Madrid) 2001; 18: 421-425.

\section{INTRODUCCIÓN}

La insuficiencia cardiaca crónica (ICC) es un importante problema de salud pública en España, con un destacado impacto asistencial y socioeconómico para el Sistema Nacional de Salud (SNS). Además, produce un importante deterioro de la calidad de vida y una disminución de la esperanza de vida en los pacientes que la padecen (1).
Aunque no se dispone con exactitud de datos epidemiológicos de la ICC en nuestro país, se estima que su prevalencia está en torno al $1 \%$ de la población general, con una incidencia anual que aumenta con la edad y que alcanza el $8,2 \%$ entre la población anciana (2).

La ICC es, en la actualidad, la primera causa de hospitalización en personas mayores de 65 años, lo que supone el $5 \%$ del total de los ingresos hospitalarios cada año (más de 75.000

Trabajo aceptado: 14 de Febrero de 2001

Correspondencia: J. Soto Alvarez. Unidad de Farmacoeconomía e Investigación de Resultados en Salud. Pharmacia, S.A. (División Searle). Avda de Burgos, 17- $1^{\text {a }}$ Planta. 28036 Madrid. 
episodios de hospitalización) (3), y las perspectivas para los próximos años son todavía más sombrías (teniendo en cuenta el progresivo envejecimiento de la población), temiéndose que aparezca lo que se ha denominado como la "epidemia de la insuficiencia cardiaca" (4).

A pesar de los avances farmacológicos de los últimos años, la mortalidad de la ICC sigue siendo muy elevada (especialmente en los casos muy avanzados), habiéndose comunicado en un estudio una mortalidad del 34\% al año, del 59\% a los 2 años, y del $76 \%$ a los 3 años (5), mientras que en otro trabajo se ha visto que el $25 \%$ de los pacientes muere al año, y aproximadamente el $66 \%$ han fallecido a los 5 años tras el diagnostico de la ICC (6). En nuestro país, se sabe que la ICC es la tercera causa de muerte por enfermedad cardiovascular (7), y la segunda causa de muerte en el número absoluto de muertes en la población general (8).

Recientemente, se ha demostrado en pacientes con ICC con disfunción sistólica del ventrículo izquierdo en clase funcional III-IV, que la asociación de espironolactona (un bloqueante de los receptores de la aldosterona) a una dosis diaria de $25 \mathrm{mg}$ a la terapia convencional de la ICC (diuréticos de asa + inhibidores de la enzima conversiva de la angiotensina (IECA) + digoxina y $\beta$-bloqueantes, ocasionalmente) produce una disminución del $30 \%$ de la mortalidad y una reducción del $35 \%$ en el número de rehospitalizaciones (9). Estos hallazgos van a suponer un incremento en la esperanza de vida para los pacientes con ICC grado funcional III-IV, y un ahorro importante en recursos sanitarios derivado del descenso en el número de episodios de hospitalización (10).

El objetivo de este estudio ha sido elaborar un análisis coste-efectividad del uso de espironolactona + terapia clásica versus placebo + terapia convencional en el tratamiento de la ICC, con el fin de conocer cual es el coste de ganar un año de vida extra en los pacientes con esta patología en grado funcional III-IV para el SNS, lo que ayudará a los agentes decisores a realizar una mejor asignación de los recursos disponibles.

\section{MATERIALES Y MÉTODOS}

Para la realización del estudio, se ha utilizado un modelo farmacoeconomico basado en un análisis de decisión, el cual va a representar el curso clínico de la ICC y la probable evolución del cuadro tras la aplicación de las alternativas terapéuticas en estudio. Cada rama del árbol de decisión que reproduce el modelo, representa una de las opciones terapéuticas evaluadas en el estudio (espironolactona y placebo) y los resultados finales de cada rama van a combinar las probabilidades de que suceda cada evento junto a los costes asociados y a los resultados clínicos finales obtenidos.

\section{A) METODOLOGÍA DE LA EVALUACIÓN ECONÓMICA}

El análisis coste-efectividad se ha realizado efectuando una simulación del comportamiento de dos hipotéticas cohortes de 1000 pacientes con ICC (una tratada con espironolactona y la otra con placebo), lo que nos permitirá conocer los desenlaces clínicos finales (efectividad) y sus costes parejos, a lo largo del tiempo medio de supervivencia de los pacientes con ICC en esta clase funcional (4,3 años) (11).

El estudio se ha realizado desde la perspectiva de la socie- dad, habiéndose incluido los costes directos médicos (medicación, días de estancia hospitalaria, pruebas complementarias efectuadas, analítica realizada y consultas médicas extras), asî como el coste de cada año de vida ganado, representado por la cuantía de la pensión media anual de nuestro país.

No se han incluido los costes indirectos (perdida de productividad por bajas laborales o ausencia al trabajo de los pacientes y/o familiares que los cuidan) dado que todos los pacientes con esta patología en clase funcional III-IV presentan incapacidad laboral permanente, y las ausencias laborales de los familiares (y la perdida de productividad asociada) sería de una cuantía muy pequeña en el coste global y además, muy difíciles de medir de forma apropiada.

Tampoco se han incluido los costes directos no médicos, como el desplazamiento de los pacientes y/o sus familiares, los honorarios de terceras personas contratadas para el cuidado del paciente en su domicilio o el valor de las horas empleadas por familiares/vecinos en cuidar a los pacientes. De la misma forma, no se han incluido tampoco los costes intangibles (derivados del sufrimiento, ansiedad, dolor, temor, etc) por ser muy difíciles de dimensionar.

Para la evaluación de los resultados se ha calculado la relación coste/efectividad de cada opción estudiada (considerándose como la más eficiente aquélla que presente un valor mas bajo), y el análisis incremental del coste y efectividad de ambas alternativas, lo que nos ha permitido conocer cual es el coste necesario para lograr una unidad extra de efectividad (año de vida ganado), derivado de usar la alternativa que presente unos mejores resultados clínicos.

El horizonte temporal del análisis ha sido de 4,3 años, por lo que se realizó un ajuste temporal, tanto de los costes como de los beneficios clínicos, habiéndose aplicado una tasa de descuento del $3 \%$.

Todas las unidades monetarias del estudio se han expresado en pesetas del año 2000.

\section{B) MEDIDA DE LA EFECTIVIDAD}

La efectividad se ha valorado como los años de vida ganados tras la administración de ambas intervenciones terapéuticas.

En la figura 1 se puede observar e árbol de decisión que representa el modelo en el que se ha basado el análisis costeefectividad. Las ramas del árbol representan la posible evolución clínica de la ICC en la práctica médica diaria, y las probabilidades de que sucedan los diferentes eventos clínicos han sido obtenidos del estudio Rales.

Los años de vida ganados con cada una de las opciones se han obtenido multiplicando los pacientes vivos en cada rama del árbol al terminar el periodo de seguimiento de Rales (2 años) por los 2,3 años de vida que cada paciente tiene de supervivencia por término medio después de la finalización de este estudio (Tabla I).

\section{C) CUANTIFICACIÓN DE LOS COSTES}

Durante los 2 primeros años de seguimiento (periodo que duró Rales) se contabilizo el número total de los episodios de hospitalización con cada rama y los costes asociados. Para el cálculo de los costes generados en cada ingreso hospitalario, se ha asumido que en cada hospitalización el paciente estaría 


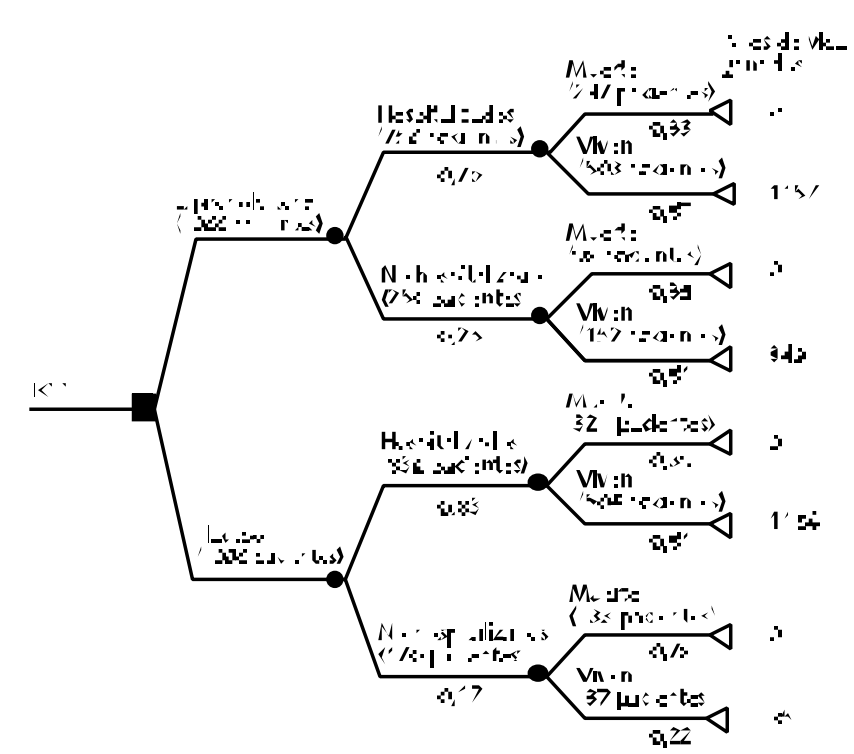

Fig. 1.

\begin{tabular}{|c|c|c|}
\hline \multicolumn{3}{|c|}{ TABLA I } \\
\hline \multicolumn{3}{|c|}{$\begin{array}{l}\text { PORCENTAJE DE SUPERVIVENCIA Y M ORTALIDAD } \\
\text { ASÍ COM O AÑOS DE VIDA GANADOS EN DOS } \\
\text { HIPOTÉTICAS CO HO RTES DE } 1.000 \text { PACIENTES CON ICC, } \\
\text { TRAS RECIBIR ESPIRO NOLACTONA O PLACEBO } \\
\text { DE ACUERDO A LOS RESULTADOS DE RALES }\end{array}$} \\
\hline & Espironolactona & Placebo \\
\hline $\begin{array}{l}\text { Hospitalización } \\
\text { M ortalidad } \\
\text { Supervivencia }\end{array}$ & $\begin{array}{l}75 \% \\
33 \% \\
67 \%\end{array}$ & $\begin{array}{l}83 \% \\
39 \% \\
61 \%\end{array}$ \\
\hline $\begin{array}{l}\text { No hospitalización } \\
\text { M ortalidad } \\
\text { Supervivencia }\end{array}$ & $\begin{array}{l}25 \% \\
39 \% \\
61 \%\end{array}$ & $\begin{array}{l}17 \% \\
78 \% \\
22 \%\end{array}$ \\
\hline $\begin{array}{l}\text { Años de vida ganados } \\
\text { Ajustados al } 3 \%\end{array}$ & $\begin{array}{l}1.506 \\
1.407\end{array}$ & $\begin{array}{l}1.249 \\
1.167\end{array}$ \\
\hline
\end{tabular}

10 días ingresado y se le realizaría 2 radiografías de tórax y 2 analíticas de rutina. Además, en un $60 \%$ de los pacientes se practicaría un ecocardiograma y todos recibirían diuréticos de asa intravenosos y dobutamina, acorde a lo publicado en nuestro medio $(12,13)$.

En el periodo posterior, los 2,3 años que resta hasta el tiempo de supervivencia medio, se asumió que cada paciente, por término medio, ingresaría cada año dos veces por descompensación de su ICC (realizándose las mismas pruebas complementarias y tratándose de igual manera que en las hospitalizaciones de la etapa anterior), acudiría 4 veces a consulta externa y se le realizarían 4 analíticas. Además, todos los pacientes estarían tratados con furosemida + un IECA + digoxina (en un $80 \%$ de los pacientes) + espironolactona de forma ambulatoria durante los años de supervivencia. Para calcular el coste futuro de los pacientes supervivientes, se multiplicó la pensión media anual de nuestro país (600.000 ptas) por los 2,3 años de supervivencia media restante.

En la tabla II, se detallan los costes de la medicación, estancia hospitalaria y demás pruebas y productos intermedios, así como la fuente de información.
TABLA II

COSTES M EDIO S (EN PESETAS) DE LOS DIFERENTES M EDICAM ENTOS, PRUEBAS COM PLEM ENTARIAS, ANALITICA Y DÍAS DE HOSPITALIZACIÓN

\begin{tabular}{lccc}
\hline Intervención & Coste/día & Rango & Fuente \\
\hline Día de hospitalización & 45.000 & $34.000-57.000$ & 14 \\
Analítica de rutina & 3.200 & $2.675-4.100$ & 14 \\
Radiografía de tórax & 2.850 & $2.550-3.238$ & 14 \\
Ecocardiograma & 22.500 & $19.500-24.750$ & 14 \\
Consulta especialista & 6.000 & $4.500-7.800$ & 14 \\
Espironolactona & 17,3 & $-\cdots-\cdots-\cdots$ & 15 \\
Diuréticos +dobutamina & 625 & $525-745$ & 15 \\
Furosemida & 16,1 & $-\cdots-\cdots--.--$ & 15 \\
IECA & 86 & $61,5-118$ & 15 \\
Digoxina & 11,5 & $9,7-11,25$ & 15 \\
\hline
\end{tabular}

\section{RESULTADOS}

Como puede apreciarse en la tabla III, el coste de la cohorte tratada con espironolactona ha sido más elevado que el del grupo tratado con placebo, con una diferencia de 141.950 ptas.

En la tabla IV queda reflejado el análisis coste-efectividad de ambas alternativas terapéuticas en evaluación, comprobándose como la relación coste/efectividad es más baja en la cohorte de espironolactona, lo que demuestra que es la opción más eficiente de las dos en estudio, al haber conseguido mucho mejores resultados clínicos con relación a los recursos consumidos. Dado que la opción de la espironolactona produce mejores resultados clínicos pero con un coste añadido, cuando se ha calculado el coste incremental de lograr un año de vida extra al

TABLA III

\begin{tabular}{|c|c|c|c|}
\hline \multicolumn{4}{|c|}{ TABLA III } \\
\hline \multicolumn{4}{|c|}{$\begin{array}{c}\text { COSTES (PTAS.) ASO CIADOS AL EM PLEO } \\
\text { DE ESPIRO NO LACTONA Y PLACEBO EN LAS DOS } \\
\text { COHORTES DE } 1.000 \text { PACIENTES, EXPRESADOS EN PESETAS } \\
\text { Y CON UN AJUSTE TEM PO RAL DEL } 3 \%\end{array}$} \\
\hline & $\begin{array}{c}\text { Costes primeros } \\
2 \text { años }\end{array}$ & $\begin{array}{c}\text { Costes los 2,3 años } \\
\text { siguientes }\end{array}$ & Costes totales \\
\hline Espironolactona & 605.625 .000 & 2.153 .804 .260 & 2.759 .429 .260 \\
\hline Placebo & 749.075 .000 & 1.868 .404 .356 & 2.617 .479 .356 \\
\hline
\end{tabular}

TABLA IV

ANÁLISIS COSTE-EFECTIVIDAD DEL USO

DE ESPIRO NO LACTO NA VERSUS PLACEBO

EN EL TRATAM IENTO DE LA ICC EN DOS HIPO TÉTICAS COHORTES DE 1.000 PACIENTES

\begin{tabular}{lcccc}
\hline & $\begin{array}{c}\text { Costes } \\
\text { (ptas) }\end{array}$ & $\begin{array}{c}\text { Años de vida } \\
\text { ganados (AVG) }\end{array}$ & Coste/AVG & $\begin{array}{c}\text { Análisis } \\
\text { incremental* }\end{array}$ \\
\hline Espirono & 2.759 .429 .260 & 1.407 & 1.961 .214 & 591.457 \\
Placebo & 2.617 .479 .356 & 1.167 & 2.242 .912 & $\cdots \cdot-\cdot-\cdots \cdot-\cdot$ \\
\hline
\end{tabular}

(*) Coste de cada año de vida ganado extra. Cálculo: coste espironolactona - coste placebo: efectividad espironolactona-efectividad placebo. 
emplear espironolactona versus placebo, se ha visto que cada año de vida ganado supone un coste adicional de 591.457 ptas.

\section{ANÁLISIS DE SENSIBILIDAD}

Con el fin de evaluar la solidez y robustez de los resultados hallados en este estudio, se realizo un análisis de sensibilidad simple univariante sobre las variables claves sometidas a mayor incertidumbre y que más podrían influir en el resultado final del análisis (16). En concreto, en este estudio se incluyeron en el análisis de sensibilidad las siguientes variables:

- rango superior e inferior del coste de cada día de hospitalización

- cambio de un $\pm 10 \%$ en el valor anual de la pensión media en nuestro país

- aumento o disminución de un año en la supervivencia media de los pacientes con ICC (5,3 y 3,3 años, respectivamente).

Como se observa en la tabla $\mathrm{V}$, la opción espironolactona presentó en todas las situaciones una mejor relación coste/efectividad cuando se comparó con placebo, objetivándose como a medida que la cifra de años de vida ganados decrece, esta relación se va aproximando cada vez más entre las dos intervenciones terapéuticas evaluadas.

\section{TABLA V}

ANÁLISIS DE SENSIBILIDAD, M O DIFICANDO LAS VARIABLES Y LOS VALORES CON MAYOR CAPACIDAD PARA INFLUIR EN LOS RESULTADOS DEL ESTUDIO

\begin{tabular}{lll}
\hline & \multicolumn{2}{c}{ Relación coste/ efectividad de ambas terapias } \\
Variables & Espironolactona & Placebo \\
\hline Precio bajo de hospitalización & 1.787 .545 & 1.910 .532 \\
$\begin{array}{l}\text { Valor alto de hospitalización } \\
\text { Un 10\% menor el valor anual }\end{array}$ & 2.108 .729 & 2.459 .982 \\
$\begin{array}{l}\text { de la pensión media } \\
\text { Un 10\% mayor el valor anual }\end{array}$ & 1.877 .529 & 2.162 .193 \\
$\begin{array}{l}\text { de la pensión media } \\
\text { Supervivencia media de los }\end{array}$ & 2.043 .739 & 2.347 .822 \\
$\begin{array}{l}\text { pacientes con ICC de 3,3 años } \\
\text { Supervivencia media de los } \\
\text { pacientes con ICC de 5,3 años }\end{array}$ & 1.602 .153 & 1.605 .985 \\
\hline
\end{tabular}

\section{DISCUSIÓN}

La ICC es un síndrome clínico con una incidencia y prevalencia en continuo ascenso en los últimos años, el cual afecta básicamente a sujetos ancianos, y que produce un elevado consumo de recursos sanitarios y una disminución significativa de la calidad de vida de los pacientes que la sufren (17).

El manejo de esta patología supone un desembolso importante de recursos para los sistemas sanitarios de los países industrializados: por encima de 10.000 millones de dólares/año en los Estados Unidos (18), alrededor de 6.800 millones de marcos/año en Alemania (19), cerca de 800.000 millones de coronas/año en Suecia (20), 360 millones de libras/año en Gran Bretaña (21), y entre 64.028 y 110.240 millones de pesetas en España (22).

El estudio Rales (9) ha demostrado que la asociación de dosis bajas de espironolactona ( $25 \mathrm{mg}$ /día) al tratamiento convencional de la ICC produce una importante y significativa reducción de la mortalidad y de la tasa de rehospitalización, habiéndose achacado, en parte, a una disminución en la aparición de arritmias ventriculares (23). El hecho de este manifiesto descenso en la hospitalización, tiene ya un considerable impacto económico directo para los SNS (24).

Los resultados de este análisis coste-efectividad demuestran que la adición de espironolactona al tratamiento clásico de la ICC va a ser una intervención terapéutica más eficiente que el uso de la terapia convencional sola, y nos va a permitir incrementar los años de vida de los pacientes. El coste secundario a cada año de vida ganado es de una pequeña cuantía (591.457 ptas.), y desde luego mucho más bajo que otros tratamientos que se administran habitualmente en la práctica asistencial cotidiana.

El estudio Rales incluyó sólo pacientes con ICC en clase funcional III-IV por disfunción sistólica del ventrículo izquierdo, por lo que los resultados de este análisis no son extrapolables a pacientes en clase funcional I y II o con ICC por disfunción diastólica, en los que el efecto de la espironolactona no ha sido aún bien evaluado.

Por otra parte, para la realización de este análisis se ha extrapolado la supervivencia media de pacientes con ICC hallada en otros entornos a nuestro país, dado que no hemos hallado datos relativos a la supervivencia media de pacientes con ICC en nuestro medio. En nuestra opinión, no debería existir una gran diferencia en las cifras, pero dado que el valor de esta variable es crucial para los resultados finales, este parámetro ha sido incluido en el análisis de sensibilidad del estudio.

La asociación de dosis bajas de espironolactona (25 mg/día) a la terapia estándar de la ICC es una opción terapéutica más eficiente que la administración de la terapia convencional únicamente, al presentar una mejor relación coste-efectividad. Por otra parte, cada año de vida extra ganado con la administración de esta asociación es de una cuantía pequeña, mucho menor que otras intervenciones terapéuticas que se aplican de forma rutinaria, y desde luego fácilmente asumible por el SNS de nuestro país.

\section{Bibliografía}

1. Sáenz de la Calzada. Insuficiencia cardiaca. Un problema de salud pública. An Med Interna (Madrid) 1999; 16: 551-2.

2. Cruz JM, López V, Gracia JC, Rojas J. Epidemiología de la insuficiencia cardiaca. Rev Esp Cardiol 1997; 50 (Spl3): 4-10.

3. Ferreira Montero IJ. Insuficiencia cardiaca: mayor morbilidad, menor mor- talidad ¿Vamos por el buen camino? Rev Esp Cardiol 2000; 53: 767-9.

4. Rodríguez F, Del Rey J. La prevención primordial y el control poblacional de la insuficiencia cardiaca. Rev Esp Cardiol 1998; 51: 977-8.

5. Cowie MR, Mosterd A, Wood DA, Reckers JW, Poole-Wilson PA, Sutton GC et al. The epidemiology of heart failure. Eur Heart J 1997; 18: 208-25. 
6. Lenni M, Tribouilloy CM, Rodeheffer RJ. Congestive heart failure in the community: trends in incidence and survival in a 10-year period. Arch Intern Med 1999; 159: 29-34.

7. Rodríguez-Artalejo F, Guallar-Castillón P, Banegas Banegas JR, del Rey Calero J. Variación geográfica en las hospitalizaciones y en la mortalidad por insuficiencia cardiaca congestiva en España, 1980-1993. Rev Esp Cardiol 2000; 53: 776-82.

8. Llisteri Caro JL, Rodríguez-Roce G, Alonso Moreno FJ, Rodríguez Padial L, Barrios Alonso V. La insuficiencia cardiaca congestiva en atención primaria (I). Semergen 2000; 26: 5-13.

9. Pitt B, Zannad F, Remme WJ, Cody R, castaigne A, Pérez A et al. The effect of spironolactone on morbidity and mortality in patients with severe heart failure. N Engl J Med 1999; 341: 709-17.

10. Azpitarte Almagro J. Los resultados del ensayo RALES constituyen una Buena noticia para los pacientes con insuficiencia cardiaca y las administraciones sanitarias. Med Clin (Barc) 2000; 114: 709-17.

11. Ho KKL, Anderson KM, Kannel WB, Grossman W, Levy D. Survival after the onset of congestive heart failure in Framingham Heart Study Subjects. Circulation 1993; 88: 107-15.

12. Navarro-López F, De Teresa E, López-Sendon JL, Castro Beiras A Guías del diagnóstico, clasificación y tratamiento de la insuficiencia cardiaca y del shock cardiogénico. Informe del grupo de trabajo de insuficiencia cardiaca de la Sociedad española de Cardiología. Rev Esp Cardiol 1999; 52 (Supl 2): 1-54.

13. Vila Alonso MT, Doce garcía MJ, Pita fernández S, Viana Zulaica C. Estudio de los casos de insuficiencia cardiaca que han requerido ingreso hospitalario. Aten Primaria 2000; 25: 78-81.
14. Base de datos de costes sanitarios. Barcelona. Soikos 2000.

15. Catálogo de Especialidades Farmacéuticas 2000; editado por el Consejo General de Colegios Oficiales de Farmacéuticos.

16. Agro KE, Bradley CA, Mittmann N, Iskedjian M, Ilersich AL, Einerson TR. Sensitivity analysis in health economic and pharmacoeconomic studies. PharmacoEconomics 1997; 11: 75-88.

17. Hoes AW, Mosterd A, Grobbee DE. An epidemic of heart failure ? Recent evidence from Europe. Eur Heart J 1998; 19 (Suppl): 2-9.

18. Karon BL. Concise review for primary-care physicians. Diagnosis and outpatient management of congestive heart failure. Mayo Clin Proc 1995; 70: 1080-5.

19. Dinkel R, Büchner K, Holtz J. Chronic heart failure . Socio-economic relevance in the Federal Republic of germany. Munch Med Weschr 1989; 131: 686-9.

20. Ryden-Bergsten T, Andersson F. The health care costs of heart failure in Sweden. J Intern Med 1999; 246: 275-84.

21. McMurray J, Hart W. The economic impact of heart failure on the UK National Health Service. Eur Heart J 1993; 14 (Supl): 133-7.

22. Antoñanzas F, Antón F, Juarez CA, Echevarria L. Costes de la insuficiencia cardiaca crónica en España. An Med Interna (Madrid) 1997; 14: 9-14.

23. Ramires FJA, Mansur A, Coelho O, Maranhao M, Gruppi CJ, Mady Ch et al. Effect of spironolactone on ventricular arrhytmias in congestive heart failure secondary to idiopathic dilated or to ischemic cardiomyopathy. Am J Cardiol 2000; 85: 1207-11.

24. Soto Alvarez J, González Vilchez F. Análisis coste-beneficio del uso de espironolactona en el tratamiento de la insuficiencia cardiaca crónica. Rev Esp Cardiol 2001; 54: 175-180. 